\title{
Changing Structures in Galactic Star Clusters
}

\author{
S. Schmeja ${ }^{1}$, M. S. N. Kumar ${ }^{1}$, D. Froebrich ${ }^{2}$ and R. S. Klessen ${ }^{3}$ \\ ${ }^{1}$ Centro de Astrofísica da Universidade do Porto, Rua das Estrelas, 4150-762 Porto, Portugal \\ email: sschmeja@astro.up.pt,nanda@astro.up.pt \\ ${ }^{2}$ Centre for Astrophysics and Planetary Science, University of Kent, \\ Canterbury, CT2 7NH, UK \\ email: df@star.kent.ac.uk \\ ${ }^{3}$ Zentrum für Astronomie der Universität Heidelberg, Institut für Theoretische Astrophysik, \\ Albert-Ueberle-Str. 2, 69120 Heidelberg, Germany \\ email: rklessen@ita.uni-heidelberg.de
}

\begin{abstract}
We investigate the structures of embedded and open clusters using statistical methods, in particular the combined parameter $\mathcal{Q}$, which permits to quantify the cluster structure. Star clusters build up from several subclusters evolving from a structured to a more centrally concentrated stage. The evolution is not only a function of time, but also of the mass of the objects. Massive stars are usually centrally concentrated, while lower-mass stars are more widespread, reflecting the effect of mass segregation. Using this method we find that in IC 348 and the Orion Nebula Cluster the spatial distribution of brown dwarfs does not follow the central clustering of stars, giving important clues to their formation mechanism by supporting the ejected embryo scenario.
\end{abstract}

Keywords. open clusters and associations: general, stars: formation, methods: statistical

\section{Introduction}

Stars are usually born in clusters embedded in the dense regions of giant molecular clouds (see Lada \& Lada 2003 for a review). The embedded phase of a cluster lasts only a few Myrs; most clusters will likely dissolve before reaching an age of 10 Myrs, less than 10 per cent of embedded clusters may survive the emergence from the parental molecular cloud to become gravitationally bound open clusters (Lada \& Lada 2003).

We investigate the structures of a number of embedded and open clusters, as well as the results of numerical simulations of clustered star formation. While the structure of an embedded cluster may hold clues to its formation mechanism, the structure of an open cluster will reflect its dynamical evolution.

There are two basic types of clusters with regard to their structure (Lada \& Lada 2003): On one hand, hierarchical clusters showing a stellar surface density distribution with multiple peaks and possible fractal substructure; on the other hand, centrally condensed clusters exhibiting highly centrally concentrated stellar surface density distributions with relatively smooth radial profiles that can be approximated by simple power-law functions or King profiles.

\section{Method}

We analyse the cluster structures using a minimum spanning tree (MST), the unique set of straight lines ("edges") connecting a given set of points without closed loops, such that the sum of the edge lengths is a minimum (e.g. Gower \& Ross 1969). Besides the 


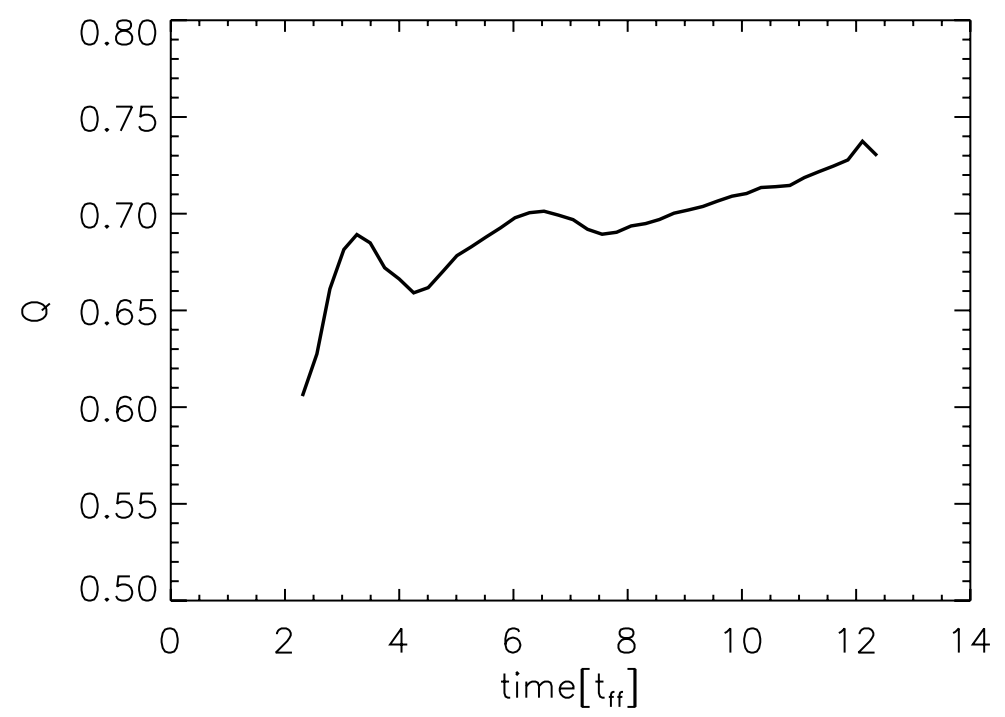

Figure 1. The temporal evolution of the clustering measure $\mathcal{Q}$ in a nascent star cluster for a numerical model with a Mach number of $\mathcal{M}=6$ and a wave number of $3 \leqslant k \leqslant 4$. The time is given in units of the free-fall time $\left(t_{\mathrm{ff}} \approx 10^{5} \mathrm{yr}\right)$.

mean edge length $\ell_{\mathrm{MST}}$ we use in particular the parameter $\mathcal{Q}$, introduced by Cartwright $\&$ Whitworth (2004) as the ratio of the normalized mean correlation length $\bar{s}$ and the normalized mean edge length $\bar{\ell}_{\mathrm{MST}}$. The dimensionless measure $\mathcal{Q}$ allows us to quantify the cluster structure and to distinguish between a centrally condensed cluster $(\mathcal{Q}>0.8)$ and a hierarchical cluster showing possible fractal substructure $(\mathcal{Q}<0.8)$. The method has been applied successfully to observed embedded clusters as well as to the results of numerical simulations (Cartwright \& Whitworth 2004; Schmeja \& Klessen 2006).

\section{Results and discussion}

\subsection{Structures changing with time}

We performed numerical SPH simulations of the fragmentation and collapse of turbulent, self-gravitating gas clouds and the resulting formation and evolution of a star cluster as described in Schmeja \& Klessen (2004). We investigated models with different turbulent velocities and driving scales and analysed the temporal evolution of the cluster structure. Although the absolute values of $\mathcal{Q}$ differ in the different models, in all models $\mathcal{Q}$ increases with time (Fig. 1), indicating that the cluster builds up from a hierarchical structure consisting of several subclusters and quickly evolves towards a more centrally concentrated state, although in no model does $\mathcal{Q}$ rise significantly above the "dividing value" of 0.8 (Schmeja \& Klessen 2006).

The analysis of near and mid-infrared data of embedded clusters in the Perseus, Ophiuchus and Serpens star-forming regions shows that the youngest Class 0 and 1 sources are usually distributed over a smaller area and show a lower $\mathcal{Q}$ value indicating a hierarchical structure possibly reflecting the fractal structure of the molecular cloud. The more evolved Class 2 and 3 objects are spread out over a larger area but show a more centrally condensed configuration in agreement with the simulations.

We also investigated a large sample of open clusters selected from the catalogue of Dias et al. (2006). Although open and embedded clusters seem to cover roughly the 

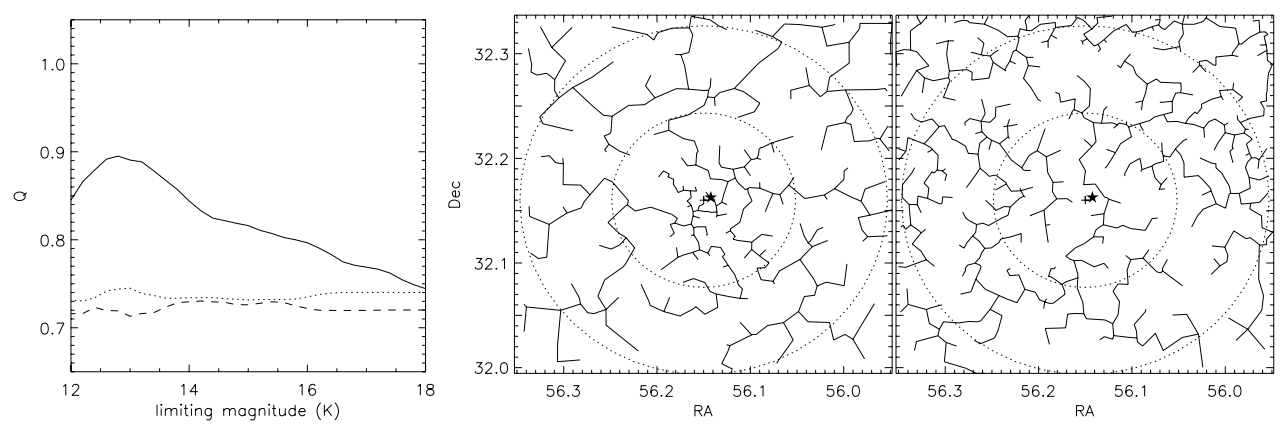

Figure 2. Embedded cluster IC 348: Changing of $\mathcal{Q}$ with the limiting magnitude ( $K$ band) for the cluster stars and two off-cluster control fields (left panel); MST of IC 348 for stars $\left(m_{K}<15\right.$; middle panel) and brown dwarfs $\left(m_{K}>15\right.$; right panel).

same range in $\mathcal{Q}$, the mean $\mathcal{Q}$ value of the sample of embedded clusters is larger than the mean $\mathcal{Q}$ value of the open clusters, and a Kolmogorov-Smirnov test suggests (with a false alarm probability $<1 \%$ ) that the $\mathcal{Q}$ values of embedded and open clusters are drawn from different populations. So we may suspect that a cluster evolving from the embedded phase to an open cluster regresses to a more hierarchical configuration due to dynamical interactions. The central condensation may be most significant in the late embedded phase, when gravity is the dominant force.

\subsection{Structures changing with stellar mass}

Almost all open and embedded clusters in our analysis show signs of mass segregation, except the youngest ones like NGC 1333 or Serpens. Considering only the brightest sources usually results in a significantly higher $\mathcal{Q}$ value. The left panel of Fig. 2 shows the dependence of $\mathcal{Q}$ on the limiting magnitude for the cluster IC 348. While the brightest (i.e. most massive) stars show a high degree of central concentration, including the faintest (substellar) objects yields a value of $\mathcal{Q} \approx 0.72$, the value expected for a random distribution.

In particular we investigate this behaviour in view of the formation of brown dwarfs. One of several theories, the so-called "ejected embryos" scenario (Reipurth \& Clarke 2001; Kroupa \& Bouvier 2003), suggests the formation of brown dwarfs as a result of premature ejection of protostellar embryos from multiple systems. Such a mechanism may be expected to produce different spatial distributions of stars and substellar objects. We analysed deep near-infrared $K$-band data obtained from the literature of the two nearby and well-sampled star-forming regions IC 348 and the Orion Trapzium Cluster. The parameters $\mathcal{Q}$ and $\ell_{\mathrm{MST}}$ are evaluated for stars and substellar objects as a function of cluster core radius. The $\ell_{\text {MST }}$ values for stars within and outside the cluster core radius vary by a factor of $\sim 2$, whereas they show roughly similar values for brown dwarfs in both IC 348 and Orion/Trapezium. So the stellar population displays a centrally concentrated distribution whereas the substellar population is distributed more homogeneously in space within twice the cluster core radius. Although the substellar objects seem to experience less influence from the cluster potential well, they are still within the limits of the cluster and not significantly displaced from their birth sites. The spatially homogeneous distribution of substellar objects with respect to stars is best explained by assuming higher initial velocities, distributed in a random manner, and going through multiple interactions in the early phase. The overall spatial coincidence of the objects with the cluster locations can be understood if these objects are nevertheless travelling slowly enough to 


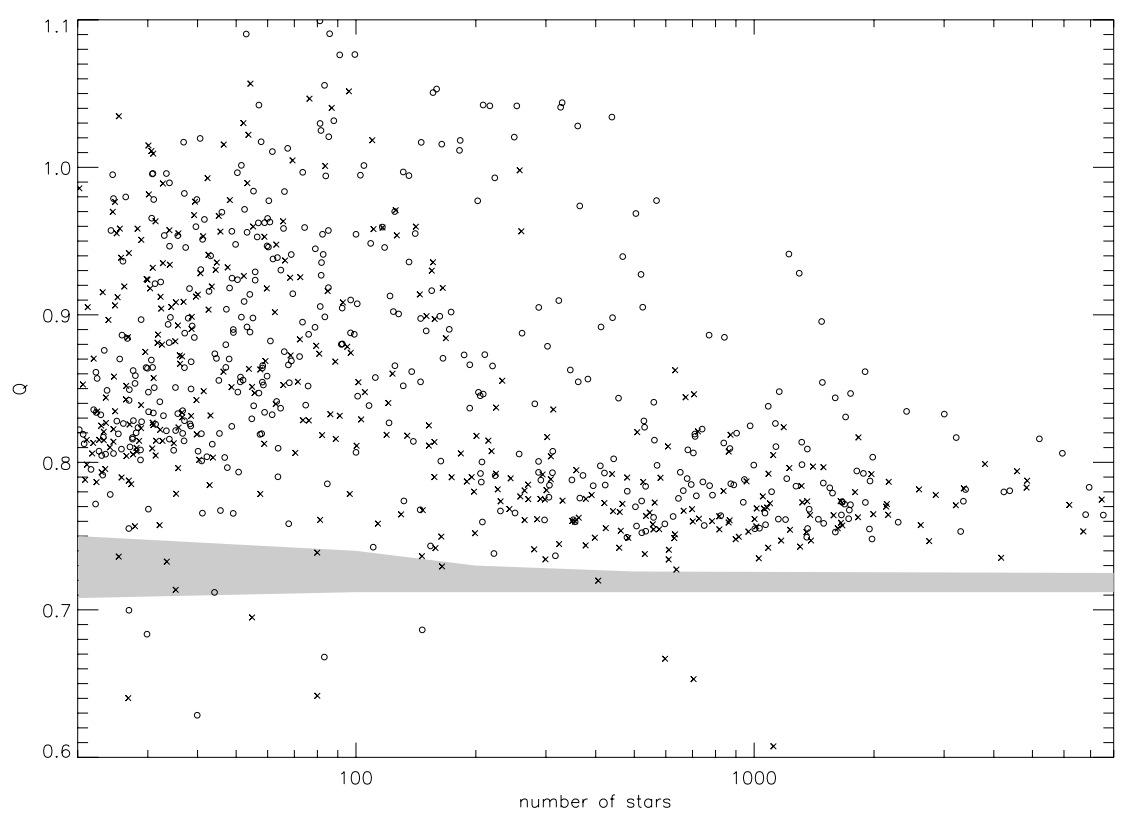

Figure 3. The $\mathcal{Q}$ parameter of known and suspected clusters plotted versus the number of objects from 2MASS data (circles: open clusters, crosses: cluster candidates). The grey-shaded area shows the parameter range expected for a random distribution of objects. Since observed globular clusters usually suffer from non-resolved central regions, thereby shifting $\mathcal{Q}$ towards lower values, they are not shown here.

feel the gravitational influence of the cluster. The observed effect is more pronounced in the older IC 348 cluster, which is in agreement with the N-body simulations of Goodwin et al. (2005) showing that a significant spatial spread of brown dwarfs with respect to stars may only occur after $5 \mathrm{Myr}$. The observational data therefore support the scenario of formation of brown dwarfs as "ejected stellar embryos". Higher ejection velocities are necessary but spatial displacements are not needed to explain the observational data. (See Kumar \& Schmeja 2007 for details.)

\subsection{Structures changing with cluster type}

Quite obviously, different types of star clusters show different structures. While globular clusters are expected to be highly centrally condensed $(\mathcal{Q} \gtrsim 1)$, open clusters are much less so. (The $\mathcal{Q}$ value of globular clusters can be very high, but in observational data it is usually limited by the membership sampling in the central region.) A random distribution, on the other hand, always produces a value around $\mathcal{Q} \approx 0.72$. We use this fact to identify and classify clusters and cluster candidates selected from 2MASS data (Froebrich et al. 2007). Fig. 3 shows the $\mathcal{Q}$ parameters of the clusters from the sample of Froebrich et al. (2007) plotted versus the number of objects: While there are many overlaps, the different cluster types clearly occupy different areas in the parameter space, which allows us to determine a probability whether a cluster candidate is a globular cluster, an open cluster or no cluster at all.

\section{Summary}

We show that the apparent structure of a star cluster strongly depends on its evolutionary stage, the limiting magnitude and the cluster type. Nascent star clusters evolve 
from a hierarchical, fractal structure with usually several subclusters to a more centrally condensed stage, which may be most pronounced while the cluster is still very young and gravity the dominant force. More evolved open clusters, on the other hand, show, in a statistical sense, less central concentration, which indicates that due to the dynamical evolution the cluster may again develop a more hierarchical structure (and eventually disperse). In almost all but the youngest clusters only the more massive stars show a significant central concentration, whereas low-mass stars and substellar objects do not follow the cluster potential and are distributed in a more homogeneous way, supporting the scenario of brown dwarfs formed as "ejected stellar embryos".

\section{Acknowledgements}

S.S. wishes to thank Annabel Cartwright and Ant Whitworth for valuable discussions. S.S. and R.S.K. acknowledge financial support from the Emmy Noether Programme of the German Science Foundation (grant KL 1358/1) for parts of this work. S.S. and M.S.N.K. are supported by a research grant POCTI/CFE-AST/55691/2004 approved by FCT and POCTI, with funds from the European community programme FEDER.

\section{References}

Cartwright, A. \& Whitworth, A. P. 2004, MNRAS 348, 589

Dias, W. S., Assafin, M., Flório, V., Alessi, B. S., \& Líbero, V. 2006, A\&̛A 446, 949

Froebrich, D., Scholz, A. \& Raftery, C. L. 2007, MNRAS 374, 399

Goodwin, S. P., Hubber, D. A., Moraux, E. \& Whitworth, A.P. 2005, Astron. Nachr. 326, 1040

Gower, J. C. \& Ross, G. J. S. 1969, Applied Statistics 18, 54

Kroupa, P. \& Bouvier, J. 2003, MNRAS 346, 369

Kumar, M. S. N. \& Schmeja, S. 2007, A\&A 471, L33

Lada, C. J. \& Lada, E. A. 2003, $A R A A 41,57$

Reipurth, B. \& Clarke, C. J. 2001, AJ 122, 432

Schmeja, S. \& Klessen, R. S. 2004, A\&A 419, 405

Schmeja, S. \& Klessen, R. S. 2006, A\&BA 449, 151 